\title{
Role of lapatinib in the first-line treatment of patients with metastatic breast cancer
}

REVIEW

\author{
This article was published in the following Dove Press journal: \\ Cancer Management and Research \\ 7 January 2010 \\ Number of times this article has been viewed
}

\author{
Catherine Oakman \\ Marta Pestrin \\ Elena Zafarana \\ Egidia Cantisani \\ Angelo Di Leo \\ "Sandro Pitigliani" Medical \\ Oncology Unit, Hospital of Prato, \\ Istituto Toscano Tumori, Piazza \\ Ospedale 2, 59100, Prato, Italy
}

Correspondence: Angelo Di Leo "Sandro Pitigliani" Medical Oncology Unit, Hospital of Prato, Istituto Toscano Tumori, Piazza Ospedale 2, 59100, Prato, Italy Tel +390574434766

Fax +390574 29798

Email adileo@usl4.toscana.it

\begin{abstract}
Lapatinib is a dual tyrosine kinase inhibitor of epidermal growth factor receptor (EGFR/ErbB1) and human epidermal growth factor receptor 2 (HER2/ErbB2). EGFR and HER2 overexpression is associated with aggressive breast cancer with a high risk of disease relapse and death. Although lapatinib targets both EGFR and HER2, its effects on HER2 appear to be more critical. The role of lapatinib in the first-line setting remains unclear. A phase II first-line monotherapy lapatinib trial in HER2-therapy-naïve metastatic breast cancer (MBC) patients confirms efficacy in HER2-positive tumors. Retrospective analysis of a phase III, first-line MBC study confirmed incremental benefit from lapatinib and paclitaxel over paclitaxel alone in HER2-positive disease. A prospective phase III study confirms superiority of letrozole and lapatinib over letrozole alone in HER2-positive MBC. Further investigation is required to define the potential first-line role for lapatinib. Particular strengths appear to be its manageable toxicity profile, lack of cross resistance with trastuzumab, activity in central nervous system disease, and synergy in combination with other anticancer therapy. Current limitations are lack of dosing recommendations from early trials, lack of predictive biomarkers beyond HER2 status, and lack of large prospective phase III trials for HER2-positive disease in the first-line setting. The role of lapatinib in HER2-negative disease is unclear.
\end{abstract}

Keywords: lapatinib, HER2, metastatic, first-line

\section{Introduction}

Metastatic breast cancer (MBC) is the leading cause of cancer death for women worldwide. Women with breast cancer that overexpresses the human epidermal growth factor receptor 2 (HER2) have increased likelihood of disease relapse and death from breast cancer.

Traditionally, cancer treatments have been trialed without identification of a specific molecular target or biomarker for prediction of response. Cytotoxic chemotherapy has focused on DNA damage and disruption of the cell cycle in rapidly dividing cells, with nonselective cell death and considerable toxicity from healthy tissue damage. Some patients experience these adverse effects for no or limited benefit. Recognition of breast cancer as a biologically heterogeneous disease and increased understanding of the molecular complexities underlying breast cancer development and progression have led to development and implementation of rationally designed targeted-therapeutics.

Potential therapeutic targets are the signaling pathways recruited by the tumor for survival and progression. It is not only the presence of drug target, but also the functional implications of blocking the target that are important. The ErbB receptors are appealing treatment targets due to their pivotal role in breast cancer.

submit your manuscript | www.dovepress.com 
ErbB 1 overexpression occurs in $20 \%$ to $30 \%$ of all primary breast cancer. ErbB2, perhaps the most studied member of the type I receptor tyrosine kinase family, is overexpressed in approximately $20 \%$ of breast cancer. Despite epidermal growth factor receptor (EGFR) overexpression and its association with worse prognosis, targeting the EGFR receptor has not shown meaningful activity in breast cancer. In contrast, therapies targeting the HER2 receptor and HER2 tyrosine kinase have shown great clinical efficacy in this tumor subtype with a poor natural history. Anti-HER2 treatments have clinical activity as monotherapy and in combination with cytotoxic agents, other targeted agents and endocrine agents. This review focuses on lapatinib, the small molecule tyrosine kinase inhibitor of both EGFR and HER2. The role of lapatinib in the first-line treatment of patients with metastatic breast cancer is, with current evidence, undefined. Review of lapatinib pharmacology, efficacy and tolerability and an overview of available lapatinib data allow us to identify lapatinib strengths and challenges, and propose its potential role in the first-line therapy of metastatic breast cancer.

\section{ErbB receptors}

The type I receptor tyrosine kinase family (ErbB) consists of 4 transmembrane receptors: ErbB1 (EGFR), ErbB2 (HER2), ErbB3 and ErbB4. ErbB2 has no exogenous ligand. ErbB3 has no kinase activity. Ligand binding of these ErbB receptors causes formation of heterodimers or homodimers, in turn inducing autophosphorylation of specific tyrosine kinase residues within conserved catalytic domains of the ErbB receptors. Tyrosine kinase autophosphorylation establishes binding sites for Src-homology 2 and phosphotyrosine binding domain containing proteins, linking ErbB to activation of downstream intracellular pathways of cell proliferation (mitogen-activated protein kinase (MAPK) pathway) and cell survival (the phosphotidylinositol-3-kinase (PI3K) pathway). ErbB2, whilst having no exogenous ligand, is the preferred partner for heterodimerization with ErbB1, ErbB3 and ErbB4 as it amplifies the mitogenic signal with potent growth and survival effects.

It is the key role of ErbB receptors in tumor cell growth and survival that make them such attractive therapeutic targets. Monoclonal antibodies, such as cetuximab and trastuzumab, target the extracellular domains of ErbB1 and ErbB2 receptors respectively. Small-molecule intracellular tyrosine kinase inhibitors of ErbB1 include erlotinib, gefitinib, and lapatinib, which also inhibits the HER2 tyrosine kinase. Targeting the EGFR receptor in breast cancer has not shown meaningful clinical activity. Conversely the efficacy of agents targeting the HER2 receptor has provided a marked improvement in the outlook for patients with HER2-positive disease.

\section{Lapatinib}

Lapatinib (GW572016) (Tykerb ${ }^{\circledR} /$ Tyverb $^{\circledR}$; GlaxoSmithKline) is an orally active, small molecule which reversibly inhibits both ErbB1 and ErbB2 (Figure 1). This concurrent inhibition in ErbB1-expressing and ErbB2-overexpressing tumors blocks the activating signaling cascades in the MAPK and PI3K pathways resulting in growth arrest and/or apoptosis, as shown in cell line and xenograft models., ${ }^{1,2}$ Preclinical models show this may be cytostatic or cytotoxic depending on cell type.

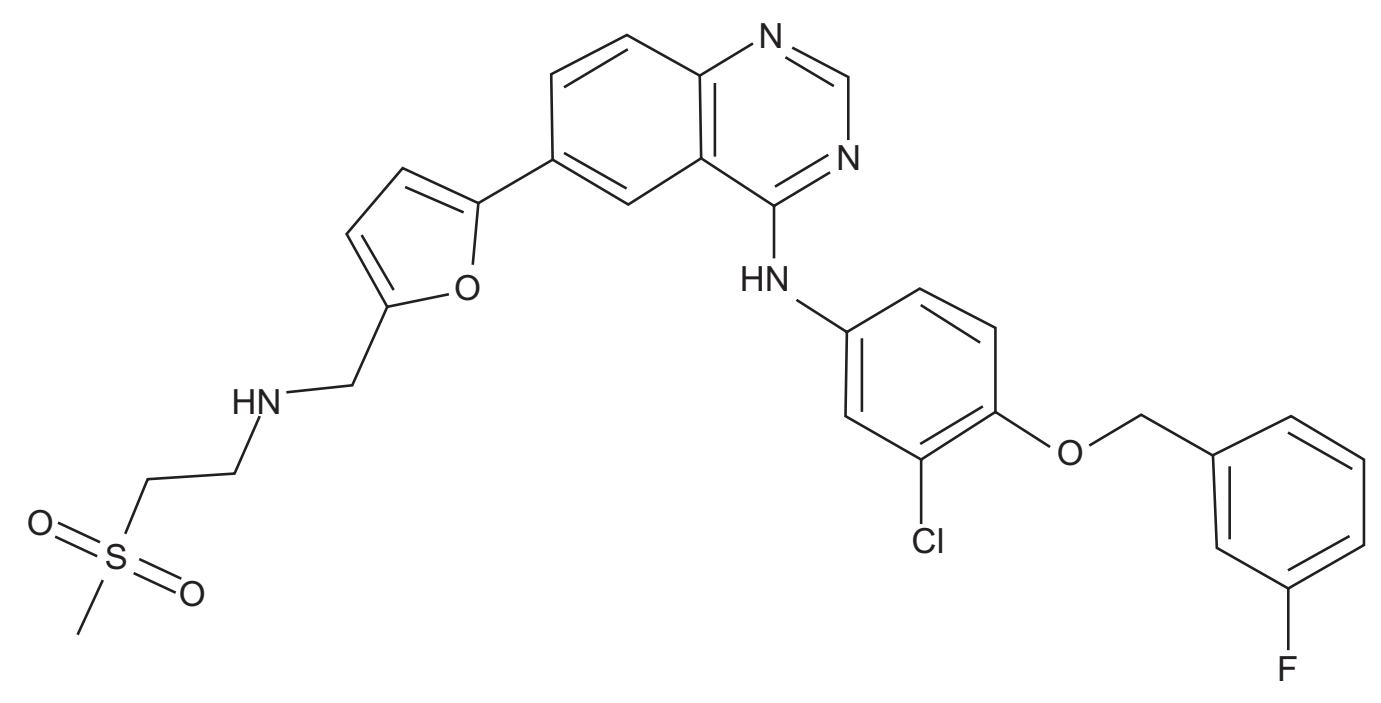

Figure I Chemical structure of lapatinib. 
The potential efficacy of lapatinib depends on the inherent biological profile of a tumor. A tumor with dependence on the EGFR and/or HER2 for cell proliferation and survival is the ideal target for lapatinib. Tumors with innate or evolved survival mechanisms which are not EGFR and/or HER2 dependent will show resistance or reduced sensitivity to therapy. Although lapatinib targets both EGFR and HER2, its effects on HER2 appear to be more critical to its efficacy.

\section{Pharmacology and pharmacokinetics}

Lapatinib was developed as an oral agent and has no intravenous equivalent. It is available as a $250 \mathrm{mg}$ tablet and currently recommended as a single dose at least 1 hour before or after a meal. ${ }^{3}$ After an oral dose, measurable lapatinib concentrations appear in the serum after 15 to 30 minutes. Pharmacokinetic (PK) studies reveal peak serum lapatinib concentrations 3 to 6 hours after dosing..$^{4-7}$ Serum concentrations increase with increased dose, although variability is high. Lapatinib displays a time dependent increase in systemic exposure with repeated dosing, with serum concentrations accumulating approximately 2 -fold with daily administration. Thus PK after the initial dose is not reflective of chronic levels. Steady state is achieved within 6 to 7 days suggesting a half-life of approximately 24 hours.

Lapatinib is highly bound to albumin $(>99 \%)$ and alpha-1 acid glycoprotein. The volume of distribution of the terminal phase of lapatinib is $>2200 \mathrm{~L}$, indicating good drug distribution. Serum concentrations of lapatinib are limited by low solubility, low permeability and extensive first pass metabolism by cytochrome P-450 enzymes CYP3A4 and CYP3A5, and to a lesser extent by CYP2C19 and CYP2C $8{ }^{7}$ One metabolite (GW690006) remains active against EGFR but not HER2. ${ }^{8}$ Less than $2 \%$ of lapatinib is excreted in the urine.

PK variations may be attributable to concurrent medications that inhibit or induce CYP3A4 or CYP3A5. Strong CYP3A4/5 inhibitors, for example azoles, antifungals, clarithromycin and grapefruit, should be avoided. If coadministration is essential, reduction in the lapatinib dose to $500 \mathrm{mg}$ once daily may appropriately compensate. ${ }^{3}$ Conversely, strong CYP3A4 inducers, eg, phenytoin, carbamazepine, dexamethasone, St. John's Wort, will reduce lapatinib serum levels and dose elevation may be required to maintain serum levels. Coadministration of lapatinib with other anticancer agents did not meaningfully alter pharmacokinetics compared with those for either agent alone. ${ }^{9-11}$

Renal impairment does not require dose alterations. Hepatic metabolism requires that severe hepatic impairment be matched by dose reduction, predicted as a reduction from
$1250 \mathrm{mg}$ once daily to $750 \mathrm{mg}$ once daily to adjust the AUC to the normal range. However there are no clinical data testing this dose adjustment. ${ }^{3}$

\section{Lapatinib dosing}

There is uncertainty about the optimal dose and schedule of lapatinib. ${ }^{12}$ In a phase I assessment of healthy volunteers, the highest administered dose was $175 \mathrm{mg}$ once daily. ${ }^{4} \mathrm{In}$ the first phase I trial to assess lapatinib dose escalation in heavily pre-treated cancer patients $(n=64)$, the highest administered dose was $1800 \mathrm{mg}$ once daily and the minimum dose with clinical activity was $650 \mathrm{mg}$ once daily. Phase I trials report responses for lapatinib monotherapy at doses ranging from 650 to $1800 \mathrm{mg}$, generally 900 to $1200 \mathrm{mg}$ daily. ${ }^{5,7}$ Lapatinib monotherapy at $500 \mathrm{mg}$ twice daily in a fasting state has been reported to have equal efficacy and toxicity to $1500 \mathrm{mg}$ once daily. ${ }^{13}$

A challenge, not only with lapatinib but also with other novel targeted therapies, is the paradigm shift away from maximum tolerated dose (MTD) to minimum effective dose. Targeted therapies, by their very nature of being 'targeted', are not associated with the systemic, dose limiting toxicities seen with traditional cytotoxic chemotherapy agents. As such, maximum administered doses in early trials may well exceed the dose required for efficacy. Although maximum tolerated or administered dose may be reported, the clinical utility of such information may be low. Incorporation of alternative endpoints for targeted agents in phase I trial design, such as pharmacokinetic and pharmacodynamic parameters, may be more useful for optimal application of results. Such endpoints may include plasma drug levels, measurement of surrogate markers for biological activity, or identification of drug target and subsequent target inhibiting dose.

\section{Lapatinib and food}

Oral administration is convenient, however attention must be given to potential sources of variability in drug exposure, particularly the effect of dosing with food which increases the bioavailability of lapatinib. In a phase I, open label trial $(n=27)$, serum drug levels were measured on 3 occasions, 1 week apart. ${ }^{14}$ For each patient, a single $1500 \mathrm{mg}$ oral dose of lapatinib was administered after a standardized high fat meal, a standardized low fat meal and in the fasting state The conditions were strict: the fasting state was dosing in the morning after an overnight fast with maintenance of the fast for 4 hours post dose; immediate dosing following a prespecified low fat breakfast ( $2 \mathrm{~g}$ fat, 520 calories); immediately following a prespecified high fat breakfast 
(54 g, 1036 calories). In comparison to the fasting state, the low fat breakfast was related to 2.67-fold increase in AUC and 2.42-fold increase in maximum concentration $\left(\mathrm{C}_{\max }\right)$. The high fat breakfast increased AUC by 4.25 -fold and $\mathrm{C}_{\max }$ by 3.03 -fold. Due to the marked variation in bioavailability, this study was practical in advising lapatinib dosing in the fasted state to achieve consistent therapeutic exposure.

Taking lapatinib with food may decrease the required dose and associated expense. However oral intake in patients with advanced malignancy is often poor and markedly variable due to disease related anorexia, nausea and vomiting, and medication side effects, especially opiate induced nausea and constipation. Dependence of lapatinib dosing on regular oral intake may be hazardous, associated with underdosing with reduced efficacy or overdosing with escalated toxicity. To advise dosing with food, it would be essential to show that food effects were reasonably consistent, with supporting pharmacokinetic and pharmacodynamic studies.

\section{Lapatinib and HER2 status}

Clinical evidence supports the use of lapatinib in HER2-positive disease. ${ }^{7,13,15-18}$ The role of lapatinib in HER2-negative disease remains unclear. ${ }^{18}$ Thus, determination of HER2 status is a critical step in optimal prescription of lapatinib. Considerable debate exists on which test represents the best assessment of HER2. Patients with a false negative result will miss potentially efficacious HER2 targeted treatment. Conversely a false positive HER2 result may expose patients to ineffective yet potentially toxic anti-HER2 therapy. Detection methods include immunohistochemistry (IHC) and fluorescence in situ hybridization (FISH). Expert guidelines have been published in an attempt to standardize assessment. The American Society of Clinical Oncology (ASCO) and the College of American Pathologists (CAP) have developed guidelines for HER2 analysis. ${ }^{19}$ The recommendations for a positive HER2 result are IHC staining of $3+$ (uniform, intense membrane staining of $>30 \%$ of invasive tumor cells), a FISH result of $>6$ HER2 gene copies per nucleus or a FISH ratio (HER2 gene signals to chromosome 17 signals) of $>2.2$. These guidelines have limitations, particularly the presumption of homogenous HER2 expression within a tumor. HER2 expression may vary within a tumor and a patient with focal HER2 amplification in a predominantly HER2-negative tumor may benefit from HER2 targeted therapy despite having HER2negative disease using the standardized criteria.

HER2 status discordance between IHC and FISH, compounded by discordance between local and central laboratories, may influence our interpretation of lapatinib trial results. For example in the phase III trial assessing capecitabine with lapatinib or placebo, trial enrolment required that patients had HER2 positive disease defined by IHC $3+$ or $2+$ with gene amplification by FISH by local institutional laboratory. ${ }^{11}$ Central analysis of archived primary tumor or biopsy of metastatic site was carried out. Of 315 patients with adequate tissue for HER2 assessment, central analysis confirmed HER2-positive status in only 241 patients $(77 \%)$.

Another issue regarding HER2 is whether to confirm HER2 status at the time of recurrent, metastatic disease. Retesting HER2 on archived tissue would allow application of current diagnostic criteria. However, a known number of false positive IHC $3+$ results are obtained when assessing HER2 status in paraffin-embedded breast cancer tissue. A biopsy from a metastatic deposit allows reassessment of disease HER2 status. Tumor cell characteristics may have changed or clonal progression to metastatic disease may have arisen from a minority population in the primary tumor. ${ }^{20} \mathrm{~A}$ potential alternative to invasive biopsy of metastatic sites is isolation and bio-characterization of circulating tumor cells (CTC). CTC analysis of HER2 status has revealed shift in HER2 status between primary tumor and metastatic disease, with either loss or gain of HER2 over expression. ${ }^{21,22}$ Systemic application of a focal biopsy result presumes biological homogeneity in the metastatic disease, which may also have limitations due to biological diversity in metastatic sites.

\section{Clinical efficacy}

Evidence for the efficacy of lapatinib in MBC derives from Phase I-III trials of monotherapy and concurrent administration of lapatinib with cytotoxic chemotherapy, other targeted treatments or endocrine agents. Most early trials were performed in patients with advanced disease, heavily pretreated with chemotherapy with or without trastuzumab. Phase I and II studies reveal good tolerance and preliminary single agent clinical activity for lapatinib monotherapy despite multiple lines of prior chemotherapy and trastuzumab, indicating lapatinib activity beyond failure of standard therapies. ${ }^{5-7,15}$ In a pivotal phase III study in MBC assessing capecitabine with or without lapatinib in trastuzumab pre-treated patients, an interim analysis of time to progression (TTP) showing superiority of the combination and lack of safety concerns led to early reporting. ${ }^{11}$ Longer median TTP was seen for capecitabine and lapatinib versus capecitabine alone (8.4 months vs 4.4 months respectively, hazard ratio [HR] $0.49 ; 95 \%$ confidence interval $[\mathrm{CI}] 0.34$ to $0.71 ; P<0.001$ ). Dual ErbB2 blockade has also been trialled in patients progressing on trastuzumab. Simultaneous inhibition of the 
ErbB2 receptor by trastuzumab, which binds the extracellular domain of ErbB2, and lapatinib, which binds the intracellular kinase, showed superiority over lapatinib therapy alone. ${ }^{10,23}$ In a phase III trial, patients $(n=296)$ receiving the combination had significantly improved progression-free survival (PFS) (12.0 weeks for the combination vs 8.4 weeks for lapatinib monotherapy; HR 0.77 ; $95 \%$ CI 0.6 to $1.0 ; P=0.029$ ) and clinical benefit rate (CBR) $(25.2$ vs $13.2 \% ; P=0.020){ }^{23}$ There was no observed difference for overall survival (OS).

In the first-line setting, although evidence is evolving it is neither extensive nor conclusive. Three published trials of upfront therapy in the metastatic setting include lapatinib monotherapy, lapatinib in combination with paclitaxel, and lapatinib plus letrozole ${ }^{13,17,18}$ (see Table 1).

\section{First-line lapatinib monotherapy}

Lapatinib monotherapy demonstrated activity as first-line therapy in HER-2 overexpressing disease in a phase II trial. ${ }^{13}$ Women $(n=138)$ with HER2-amplified locally advanced or $\mathrm{MBC}$ were randomized to one of two schedules of lapatinib monotherapy: $500 \mathrm{mg}$ twice daily or $1500 \mathrm{mg}$ once daily. Background PK data suggested that the $500 \mathrm{mg}$ twice daily AUC would be greater and that plasma drug levels would vary less with twice daily dosing. ${ }^{24}$ Equal efficacy for the two schedules was reported. Objective response rate (ORR) was $24 \%$ (1500 mg once daily $22 \%, 500 \mathrm{mg}$ twice daily $26 \%, P=0.691)$ - comparable to first-line trastuzumab therapy ${ }^{25,26}$ - and the CBR was 31\% (1500 mg once daily $29 \%, 500 \mathrm{mg}$ twice daily $33 \%, P=0.714$ ). Median time to response was 7.9 weeks. Median duration of response was 28.4 weeks, indicating long term benefit from lapatinib. Of note, no patients had received prior trastuzumab and only $50 \%$ had received any adjuvant or neoadjuvant systemic therapy. In contrast, most patients now presenting with HER2-positive advanced disease will have received adjuvant chemotherapy and trastuzumab. The first-line activity of lapatinib monotherapy in a more representative population is unknown. These results do suggest that in women presenting with MBC, with no prior HER2 treatment, lapatinib monotherapy may be a reasonable option for first-line treatment.

\section{First-line lapatinib and chemotherapy}

As with other targeted agents, combination therapy with lapatinib may be the best clinical approach for efficacy and duration of response. Paclitaxel is a microtubule damaging agent with proven activity in breast cancer. Phase I data

Table I First-line lapatinib trials in metastatic breast cancer

\begin{tabular}{|c|c|c|c|c|c|}
\hline Reference & Trial & Population & Pts & Therapy & Outcomes \\
\hline Gomez et $\mathrm{al}^{13}$ & $\begin{array}{l}\text { Phase II } \\
\text { Multicenter, randomized, } \\
\text { open label }\end{array}$ & $\begin{array}{l}\text { HER2-positive } \\
\text { advanced or MBC }\end{array}$ & 138 & $\begin{array}{l}\text { Lapatinib } 500 \mathrm{mg} \text { twice daily } \\
(\mathrm{n}=69) \\
\text { vs } \\
\text { Lapatinib I } 500 \mathrm{mg} \text { once daily } \\
(\mathrm{n}=69)\end{array}$ & $\begin{array}{l}\text { ORR } 24 \% \\
\text { CBR } 31 \% \\
\text { PFS } 16.1 \text { wk } \\
\text { Equal efficacy between doses }\end{array}$ \\
\hline Di Leo et al $^{17}$ & $\begin{array}{l}\text { Phase III } \\
\text { Multicenter, randomized, } \\
\text { double-blind, placebo- } \\
\text { controlled }\end{array}$ & $\begin{array}{l}\text { HER2-negative or } \\
\text { unknown advanced } \\
\text { or MBC }\end{array}$ & 579 & $\begin{array}{l}\text { Lapatinib I } 500 \mathrm{mg} \text { once daily } \\
\text { plus paclitaxel } 175 \mathrm{mg} / \mathrm{m}^{2} \text { iv } \\
\text { every } 2 \text { I days }(\mathrm{n}=29 \mathrm{I}) \\
\text { vs } \\
\text { Placebo plus paclitaxel } \\
\mid 75 \mathrm{mg} / \mathrm{m}^{2} \text { iv every } 2 \text { I days } \\
(\mathrm{n}=288)\end{array}$ & $\begin{array}{l}\text { ITT population: } \\
\text { ORR } 35.1 \% \text { vs } 25.3 \% P=0.008 \\
\text { CBR } 40.5 \% \text { vs } 31.9 \% P=0.025 \\
\text { EFS } 25.1 \text { wk vs } 22.6 \text { wk } P=0.238 \\
\text { HER2-positive ( } n=86 \text { ): } \\
\text { (retrospective analysis) } \\
\text { ORR } 63.3 \% \text { vs } 37.8 \% P=0.023 \\
\text { CBR } 69.4 \% \text { vs } 40.5 \% P=0.011 \\
\text { EFS } 35.1 \text { wk vs } 21.9 \text { wk } P=0.004\end{array}$ \\
\hline Johnston et al ${ }^{18}$ & $\begin{array}{l}\text { Phase III } \\
\text { Multicenter, randomized, } \\
\text { double-blind, placebo } \\
\text { control }\end{array}$ & $\begin{array}{l}\text { HR-positive MBC } \\
\text { in postmenopausal } \\
\text { women }\end{array}$ & 1286 & $\begin{array}{l}\text { Lapatinib I } 500 \mathrm{mg} \text { once daily } \\
\text { plus letrozole } 2.5 \mathrm{mg} \text { once daily } \\
\text { ( } \mathrm{n}=642) \\
\text { vs } \\
\text { Placebo plus letrozole } 2.5 \mathrm{mg} \\
\text { once daily }(\mathrm{n}=644)\end{array}$ & $\begin{array}{l}\text { ITT population: } \\
\text { ORR } 33 \% \text { vs } 32 \% P=0.726 \\
\text { CBR } 58 \% \text { vs } 56 \% P=0.76 \mathrm{I} \\
\text { PFS } 11.9 \text { mo vs } 10.8 \mathrm{mo}, P=0.026 \\
\text { HER2-positive pts }(\mathrm{n}=219) \text { : } \\
\text { ORR } 28 \% \text { vs I5\%, } P=0.021 \\
\text { CBR } 48 \% \text { vs } 29 \%, P=0.003 \\
\text { PFS } 8.2 \text { mo vs } 3 \mathrm{mo}, P=0.019\end{array}$ \\
\hline
\end{tabular}

Abbreviations: $C B R$, clinical benefit rate; $E F S$, event-free survival; $H R$, hormone receptor; ITT, intent to treat; IV, intravenous; MBC, metastatic breast cancer; MO, months; ORR, objective response rate; PFS, progression-free survival; Pts, patients;WK, weeks. 
from patients with refractory disease $(n=26)$ supported the use of combination paclitaxel and lapatinib, with objective responses (OR) in 3 patients with taxane resistant $\mathrm{MBC}$ and stable disease $(\mathrm{SD})>12$ weeks in 7 patients. ${ }^{27}$

A large multicenter, randomized, double-blind phase III trial assessed the combination of lapatinib and paclitaxel in the first-line metastatic setting. ${ }^{17}$ A primary goal of this trial was to explore the efficacy of lapatinib in women with advanced tumors without HER2 amplification/overexpression. Women ( $\mathrm{n}=579$ ) with HER2-negative or HER2-uncharacterized MBC were randomized to paclitaxel $175 \mathrm{mg} / \mathrm{m}^{2}$ iv every 21 days plus lapatinib $1500 \mathrm{mg}$ once daily or placebo. OR were reported in $35 \%$ and $25.3 \%$ of patients in the paclitaxel-lapatinib arm and paclitaxel-placebo arm, respectively $(P=0.008)$. However, there was no significant difference in TTP or OS between the treatment arms. From a preplanned, retrospective, blinded, central analysis of tumor tissue for HER2 using FISH and IHC, 86 patients were found to have HER2-positive disease. For patients with both FISH and IHC, there was, as expected, a strong association between HER2 gene amplification and HER2 protein overexpression. For the HER2-negative patients, no differences were seen for any outcome. However, for the HER2-positive minority, treatment with lapatinib/paclitaxel resulted in statistically significant improvements in TTP, event-free survival (EFS), ORR and CBR. No significant OS benefit was reported. These patients are small in number and were not randomized by HER2 status, but they were well balanced between the treatment groups. Median TTP for the paclitaxel/lapatinib-treated HER2-postive patients was 36.4 weeks compared with 25.1 weeks in the paclitaxel/placebo-treated patients (unadjusted HR of 0.53 ; $95 \%$ CI 0.31 to $0.89 ; P=0.005)$. In the paclitaxel/lapatinib versus the paclitaxel/placebo treated patients, ORR $(63.3 \%$ vs $37.8 \%$ respectively, $P=0.023)$ and CBR $(69.4 \%$ vs $40.5 \%$, respectively, $P=0.011$ ) were significantly higher. Thus, additional benefit from lapatinib was reported only in women with HER2-amplified disease, indicating that lapatinib exerts its primary effects through inhibiting the HER2 pathway. Despite lapatinib being a dual kinase inhibitor, EGFR did not show any correlation with clinical efficacy. These preliminary, hypothesis generating results require prospective confirmation. A current trial is prospectively assessing first-line lapatinib and weekly paclitaxel $80 \mathrm{mg} / \mathrm{m}^{2}$ in HER2-positive MBC.

\section{First-line lapatinib and endocrine agents}

Despite documentation of HER2-positive and hormone receptor (HR)-positive status in $\mathrm{MBC}$, many patients will display resistance to anti-HER2 therapy and/or endocrine therapy. A potential mechanism of resistance is downstream crosstalk between ErbB2 and HR signaling pathways. Dual blockade of HER2 and HR may overcome this crosstalk and improve outcomes. In the endocrine treatment of HR-positive HER2-positive tumors, in which overexpression of HER2 may confer resistance to endocrine therapy, concurrent inhibition of HR and ErbB2 may enhance efficacy. In addition, in HR-positive HER2-negative tumors, the early use of ErbB inhibitors may prevent or limit the upregulation of ErbB pathways that often occurs in the progression of disease. ${ }^{28}$ To this ends, several targeted agents are being investigated in combination with endocrine therapy. ${ }^{29-31}$ Trastuzumab plus anastrazole has shown improved PFS over endocrine blockade alone in women with HR-positive HER-2 positive $\mathrm{MBC}$, and gefitinib plus anastrazole was superior to anastrazole alone in HR-positive MBC patients. ${ }^{29,30}$

A recently reported phase III trial randomized post menopausal women with HR-positive MBC $(n=1286)$ to the nonsteroidal aromatase inhibitor letrozole $2.5 \mathrm{mg}$ once daily plus placebo or lapatinib $1500 \mathrm{mg}$ once daily as first-line therapy. ${ }^{18}$ Prior neoadjuvant/adjuvant antiestrogen therapy was allowed, as were adjuvant aromatase inhibitors and trastuzumab if discontinued $>12$ months prior to trial entry. In women with HR-positive HER2-positive disease $(n=219)$, after a median follow up of 1.8 years, the combination of letrozole-lapatinib was superior to letrozole alone, with median PFS of 8.2 and 3.0 months, respectively ( $\mathrm{HR}=0.71,95 \%$ CI 0.53 to 0.96 , $P=0.019)$. Lapatinib-letrozole also improved CBR (48\% vs $29 \%$ ). There was no significant improvement in OS, however less than $50 \%$ of OS events had occurred at time of reporting. Patients with HR-positive HER2-negative disease $(n=952)$ had no improvement in PFS. Within this subgroup, patients who were endocrine treatment naïve or had not received endocrine therapy for $\geq 6$ months (after a median exposure to tamoxifen of 5 years) had no additional benefit from the combination over letrozole alone. In contrast, in women with $\leq 6$ months since discontinuation of adjuvant tamoxifen therapy, a nonsignificant trend was reported toward prolonged PFS $(\mathrm{HR}=0.78 ; 95 \%$ CI 0.57 to $1.07 ; P=0.117)$. In this group, adjuvant tamoxifen had been administered for a median of only 2.8 years, suggesting relative tamoxifen resistance, and median time since discontinuation was only 1 month. Thus, within the HER2-negative population, targeting the EGFR/HER2 receptor may add benefit and may reflect a more prominent role played by growth factor signaling. Lack of PR expression has been postulated as a surrogate for endocrine resistance. This trial supports the use 
of combined therapy of letrozole and lapatinib in patients with HR-positive HER2-positive disease over letrozole alone. Within the HER2-negative HR-positive patients, no clinically meaningful results reached statistical significance, however further trials assessing biomarkers and stratification based on prior responsiveness to endocrine therapy may uncover a subgroup with benefit.

\section{Role of lapatinib in CNS metastases}

First-line lapatinib, either alone or in combination with radiation, surgery or other anticancer treatment, for patients with central nervous system (CNS) metastases has not been prospectively explored. Observations from the phase II monotherapy study were of 6 patients with stable CNS disease at study entry, 1 patient had CNS disease as the sole site of disease progression, 3 patients had systemic progression only, 1 patient died before documented progression and 1 patient continued progression free at time of discontinuation. ${ }^{13}$ Although first-line data are currently lacking, CNS activity of lapatinib may be a strength in defining its place in therapy.

The CNS remains a concerning site for initial and subsequent relapse for patients with HER2-positive breast cancer. This may represent an innate tendency of HER2-positive tumors to infiltrate the CNS, improved systemic control with trastuzumab with longer survival time to develop CNS metastases and/or the CNS as a sanctuary site for metastases due to poor trastuzumab penetrance across the blood-brain barrier (BBB). Monoclonal antibodies are prevented from entering the CNS efficiently, predominantly due to their large size. In preclinical models, lapatinib did not cross the $\mathrm{BBB}$ to a significant degree. However in CNS disease, the disturbed BBB may have altered permeability, allowing passage of lapatinib. In a preclinical model, lapatinib was shown to inhibit the formation of brain metastases in a breast cancer xenograft mode. ${ }^{32}$ In this murine model, lapatinib inhibited phosphorylation of EGFR, HER2 and associated downstream proteins. Of note, lapatinib inhibited formation of large metastases but did not completely prevent metastases, suggesting resistance in some breast cancer cells.

Lapatinib activity in CNS disease in heavily pre-treated patients encourages further research into defining its role in the management of CNS metastases. In the phase III trial with lapatinib and capecitabine, fewer patients had CNS metastases in the combination group $(n=4)$ vs lapatinib monotherapy ( $\mathrm{n}=11)$, however this was not statistically significant. ${ }^{11}$ A phase II analysis of lapatinib in MBC patients $(n=39)$ with new or progressive brain metastases following trastuzumab therapy revealed 1 patient with a PR and 7 patients with stable
CNS and non-CNS disease at 16 weeks. ${ }^{33}$ Within this trial, an exploratory analysis using volumetric assessment rather than RECIST for CNS lesions suggested longer TTP in patients with $>10 \%$ volumetric reductions. Whilst this study did not reach its primary efficacy goal for response rate based on the prospectively defined RECIST criteria, the volumetric studies were certainly encouraging. Preliminary data from a subsequent trial $(\mathrm{n}=104)$, with volumetric reduction of CNS lesions as its primary endpoint, revealed $\geq 20 \%$ volumetric reduction for 17 patients $(16.3 \%)$ in whom the median time to volume progression was 16 weeks (range 12 to 24 weeks). ${ }^{34}$ Definitive results are awaited. Furthermore, a trial extension offered patients with CNS and/or non-CNS progression on lapatinib alone the option of receiving combination lapatinib and capecitabine. ${ }^{35}$ Preliminary results $(\mathrm{n}=40)$ showed $\geq 50 \%$ reduction in 8 patients $(20 \%)$ suggesting activity of lapatinib-capecitabine beyond lapatinib monotherapy resistance.

\section{Lapatinib tolerability}

Lapatinib is a generally well tolerated treatment. In phase I and II studies, lapatinib monotherapy was associated with transient grade 1-2 rash, diarrhea, nausea/vomiting, stomatitis, fatigue and anorexia reported as the most frequent adverse events (AE). ${ }^{4-7,13,15,16}$ Grade 3 toxicities were uncommon, but included diarrhea, rash, abnormal liver function and gastrointestinal events. ${ }^{5-7}$ No grade 4 toxicity attributable to lapatinib was reported..$^{5-7,13,16}$ No drug-related cardiac toxicity was observed. This seems to be in contrast to the reversible cardiomyopathy seen with trastuzumab therapy, although no direct comparison data are available. There was no drugrelated interstitial pneumonitis.

\section{Diarrhea}

Lapatinib associated diarrhea correlates with dose but not with serum concentration. ${ }^{7}$ This suggests that the diarrhea is due to a local effect on the gut epithelium. The issue about drug dosing may be pertinent to diarrhea as it is dose dependent. Lower doses due to administration with food or twice daily scheduling may be associated with less diarrhea. A pooled analysis of 9 phase I-III clinical trials was undertaken to review diarrhea associated with lapatinib as monotherapy or in combination with capecitabine or taxanes. ${ }^{36}$ Lapatinib doses ranged from 1000 to $1500 \mathrm{mg}$ once daily. Diarrhea occurred in 55\% of lapatinib-treated patients and $24 \%$ of patients not receiving lapatinib. Overall, most diarrhea events were grade 1-2, self-limiting, and manageable with conventional approaches. Grade 3 events occurred 
in $<10 \%$ of patients and grade 4 events were rare $(\leq 1 \%)$. Dose modifications were seldom required. Diarrhea was generally an early event with onset within 6 days of commencing treatment, with a median duration of 7 to 9 days. Although the elderly ( $>70$ years) patient population was small, the incidence of diarrhea events was comparable to that observed in younger patients. Proactive management of diarrhea, with early implementation of antidiarrhea agents and increased fluid, is a crucial component of lapatinib prescription.

\section{Rash}

Lapatinib rash is generally mild. ${ }^{7}$ Interestingly, despite a correlation between rash and efficacy for other EGFR inhibitors, no such correlation is seen for lapatinib. Rash has been reported to be more prevalent in nonresponders. ${ }^{7}$ Structurally lapatinib, a 4-anilinoquinazoline, differs from erlotinib and gefitinib, quinazolines, which may account for the difference in rash. In an early study, the rash was independent of serum concentration, appeared between 2 and 66 days, generally resolved without interruption of treatment and seemed resistant to topical dermatological therapy. ${ }^{7}$ A pooled analysis of dermatological events from lapatinib at doses from 1000 to $1500 \mathrm{mg}$ once daily in patients $(n=1419)$ from 9 clinical trials of metastatic cancer was also reported. ${ }^{37}$ Lapatinib was administered as monotherapy or in combination with capecitabine or paclitaxel. Events included hand-foot syndrome, rash, hair disorder, dry skin, pruritus/urticaria, skin disorder, skin infection, and nail disorder. Lapatinib monotherapy was associated with events in $58 \%$ of patients: $55 \%$ grade $1 / 2,3 \%$ grade 3 , no grade 4 . The most common event was rash (43\%). Most events develop early, between days 1 and 14 of starting treatment, with a median duration of 29 days. Adverse skin events infrequently required lapatinib dose reduction (3\%), dose interruption (7\%) and drug discontinuation (1\%).

\section{Cardiotoxicity}

Although cardiotoxicity with decreases in left ventricular ejection fraction (LVEF) is reported with trastuzumab therapy, lapatinib cardiotoxicity is uncommon. In a review of cardiac safety in lapatinib treated patients, 1.3\% (42 of 1327) experienced a decrease in LVEF, 23 of whom received monotherapy and 19 of whom received it in combination with other chemotherapeutic agents. ${ }^{38}$ Only 4 of the 42 patients were symptomatic $(0.01 \%)$ and they responded to standard heart failure treatment. Decreased LVEF occurred within 9 weeks of treatment initiation in $69 \%$ of cases and resolved in $62 \%$ of patients. $90 \%$ had confounding factors: prior exposure to adriamycin/cyclophosphamide, radiotherapy or trastuzumab. Even in combination with trastuzumab, cardiotoxicity is uncommon. ${ }^{10,23}$

\section{Specific toxicity from first-line lapatinib trials}

\section{First-line lapatinib monotherapy}

In the phase II monotherapy study, AEs considered related to lapatinib occurred in $71 \%$ of patients. ${ }^{13}$ The most common AEs were grade 1-2 diarrhea, rash, pruritis and nausea. Grade 3 events were diarrhea (3\%), rash (1\%) and nausea (1\%). Serious AEs (SAEs) attributed to lapatinib were experienced by $7 \%$ of patients. Four patients permanently withdrew from the study due to lapatinib related SAEs; grade 3 abnormal hepatic function, grade 1 left ventricular dysfunction, grade 2 ejection fraction decrease and grade 4 thrombocytopenia with grade 3 anemia in 1 patient. Six fatal AEs were reported, only 1 considered related to lapatinib: a 73-year-old patient with hepatic failure and bacterial peritonitis, on a background of 223 days of $500 \mathrm{mg}$ oral twice daily lapatinib for extensive liver metastases.

\section{First-line lapatinib plus paclitaxel}

In keeping with the known side effects of both drugs, common side effects from paclitaxel and lapatinib were diarrhea, rash, alopecia, nausea, vomiting, myalgia and neutropenia, all generally mild. ${ }^{17}$ The combination was associated with significantly greater toxicity, especially diarrhea and rash. The addition of lapatinib resulted in increased grade 3 rash (4\% vs $0 \%$ ) and grade 3 diarrhea ( $15 \%$ vs $1 \%$ ). Dose reductions to $1250 \mathrm{mg}$ once daily in $6 \%$ patients and to $1000 \mathrm{mg}$ once daily in $<1 \%$ patients were required for toxicity management. AEs resulted in treatment discontinuation in $16 \%$ and $7 \%$ of patients receiving paclitaxel/lapatinib and paclitaxel/placebo respectively. Cardiac events were reported in 6 patients in each of the treatment groups. In 5 of each group of 6 , this decrease in LVEF was asymptomatic. There were $8(2.7 \%)$ $\mathrm{SAE}$ related deaths in the paclitaxel/lapatinib arm and $2(0.6 \%)$ in the paclitaxel/placebo arm. These fatal AEs in the paclitaxel/lapatinib arm were due to septic shock and diarrhea (3 patients), septic shock, cerebrovascular accident, pulmonary embolism, cardiac arrest and heart failure. The cardiac arrest and heart failure were not considered treatment related. In the paclitaxel-placebo arm, the deaths were due to a cerebrovascular accident and an unknown cause.

\section{First-line lapatinib plus letrozole}

Common side effects were diarrhea, rash, nausea, arthralgia and fatigue. ${ }^{18}$ Toxicity was greater in the lapatinib-letrozole 
arm compared with letrozole-placebo arm, particularly with grade 3 or 4 diarrhea (10\% vs $1 \%$ respectively) and rash ( $1 \%$ vs $0 \%$ respectively). Of the 60 patients with grade 3 or 4 diarrhea in the combination arm, 15\% required discontinuation and $19 \%$ required a dose reduction. Cardiac toxicity was infrequent with 7 patients having symptomatic LVEF decline, 2 from the letrozole/placebo arm and 5 from the letrozole/lapatinib arm. Treatment related liver toxicity was reported in 1 patient from the letrozole/placebo arm and 8 patients from the letrozole-lapatinib arm, 2 of whom required drug discontinuation, with subsequent resolution of liver function. SAEs occurred in $8 \%$ of patients in the combination arm and $4 \%$ in the letrozole-placebo group. There were 8 deaths in each treatment arm. One death from hepatobiliary toxicity in the letrozole/lapatinib arm, and 2 deaths in the letrozole-placebo arm were considered SAE related to study drug. Use of the drugs in combination did not reveal any new safety concerns for either drug.

\section{Lapatinib resistance}

A strength of lapatinib is its noncross resistance with trastuzumab. Clinical responses are seen with lapatinib, even in HER2-positive MBC patients pretreated with 1 or more lines of prior trastuzumab. This lack of cross resistance between trastuzumab and lapatinib suggests diverse mechanisms underlying the resistance.

Despite documented HER2 receptor amplification, patients may have de novo or acquired resistance to trastuzumab. ${ }^{39}$ Potential mechanisms of trastuzumab resistance include altered receptor-antibody interaction (MUC4, p95 ${ }^{\text {HER2 }}$, ErbB2 mutations), altered downstream signaling (reduced p27, reduced PTEN, activation of PI3K, activation of Akt) and crosstalk with other signaling pathways (IGF-R1, ER, VEGFR). ${ }^{40}$ The p95 ${ }^{\mathrm{HER} 2}$ receptor is created either by cleavage and shedding of the extracellular domain of the HER2 receptor or by specific mRNA splicing, resulting in a constitutively active truncated receptor which is associated with a more aggressive phenotype. The intracellular mechanism of action of lapatinib, in contrast to the extracellular approach of trastuzumab, results in inhibition the phosphorylation of $\mathrm{p} 95^{\mathrm{HER} 2}{ }^{41}$ PTEN reduction or deficiency results in increased signaling via the critical $\mathrm{PI} 3 \mathrm{~K} /$ Akt pathway. PTEN loss is associated with a lower response to trastuzumab, however lapatinib appears PTEN independent and seems to maintain activity despite loss of this tumor suppressor. ${ }^{42,43}$

The efficacy of lapatinib is also limited by resistance. ${ }^{44,45}$ This may be mediated by activation of redundant survival pathways, rather than ErbB2 receptor mutations. A preclinical breast cancer cell model showed outgrowth of cells with acquired resistance to lapatinib with prolonged exposure, despite initial high sensitivity. ${ }^{45}$ Resistance was not associated with reduced inhibition of the HER2 pathway, but it was associated with increased survivin. Prolonged inhibition of ErbB2 kinase activity resulted in upregulation of the transcription factor FOXO3A which upregulates estrogen receptor (ER) expression and signaling. Regulation of survivin and tumor cell survival switched from ErbB2 alone to ErbB2 and other pathways. The lapatinib resistant cells do not entirely abandon the HER2 pathway. Instead they develop codependence between HER2 and ER pathways.

\section{Quality of life}

In the setting of advanced disease, the intention of treatment is palliative. An essential component of caring for individuals with advanced disease is to improve or maintain quality of life (QoL) and to minimize pain. The common QoL issues to consider are disease induced pain, immobility, anxiety, anorexia and fatigue, and treatment related side effects. In combination these effects are potentially profoundly disabling in terms of independence, physical activity and social functioning.

Lapatinib is an oral treatment so obviates the need for intravenous access or oncology day visits for therapy administration. Lapatinib is commonly reported as well tolerated with mild and manageable side effects. That the treatment is well tolerated is indicated by $\geq 80 \%$ compliance. ${ }^{16}$ About $25 \%$ of patients require dose adjustment and/or treatment interruption due to AEs. ${ }^{15}$ The commonest AEs may significantly impair QoL, despite being mild or moderate. Depending in the distribution and severity of rash, this can impair personal and social functioning. Diarrhea can be disabling, even at grade 2 (see Table 2). Particularly with the diarrhea, proactive management may result in reduced incidence and severity, and less impact on QoL.

Published QoL data for lapatinib comes from its combination use with capecitabine. ${ }^{46} \mathrm{~A}$ QoL analysis was undertaken in the study of capecitabine monotherapy versus capecitabine and lapatinib using the validated Functional Assessment of Cancer Therapy-Breast (FACT-B) and EuroQoL (EQ-5D) questionnaires. QoL for patients in both treatment groups was maintained, with a suggestion of improved QoL for those with combination therapy. In an exploratory analysis, mean changes from baseline in all QOL scores were similar for both groups. Patients with 
Table 2 National Cancer Institute Common Toxicity Criteria (NCl-CTC) grading for diarrhea

\begin{tabular}{ll}
$\begin{array}{l}\text { Toxicity } \\
\text { grade }\end{array}$ & Diarrhea \\
\hline I & $\begin{array}{l}\text { Increase of }<4 \text { stools/day over baseline } \\
\text { Mild increase in ostomy output compared with baseline }\end{array}$ \\
2 & $\begin{array}{l}\text { Increase of } 4-6 \text { stools/day over baseline } \\
\text { Intravenous fluids }>24 \mathrm{~h} \\
\text { Moderate increase in ostomy output compared with } \\
\text { baseline }\end{array}$ \\
& Not interfering with daily living \\
& $\begin{array}{l}\text { Increase of }>7 \text { stools/day over baseline } \\
\text { Incontinence } \\
\text { Intravenous fluids } \\
\text { Severe increase in ostomy output compared with baseline } \\
\text { Interfering with daily living activities } \\
\text { Life-threatening consequences (eg, hemodynamic collapse) } \\
\text { Death }\end{array}$ \\
\hline
\end{tabular}

an OR or SD showed clinically meaningful differences in QoL scores compared with patients with progressive disease. The challenge of this QoL study however is that lapatinib versus placebo was not assessed. The comparison was between lapatinib plus capecitabine and capecitabine alone, which is itself related to significant disability from diarrhea, nausea and rash.

\section{Biomarkers}

The use of targeted therapies is likely to be critically guided by patient selection and predictive molecular markers. Due to biological heterogeneity within $\mathrm{MBC}$, some patients will be more likely to benefit from a particular intervention. The ideal of individualized anticancer therapy requires predictive biomarkers to firstly, identify which patients will benefit, and secondly, monitor response. The importance of such markers in trial design and in the assessment of efficacy of targeted therapies should not be overlooked.

Preclinical and clinical studies support HER2 amplification as predictive of response to lapatinib. ${ }^{5-7,15,17,47}$ In contrast, this differential benefit is not evident in patients with HER2-negative status, even when HER2 status is centrally confirmed. ${ }^{47}$ Whilst HER2 amplification identifies patients who are likely to respond, resistance within this population is frequent and HER2 status alone will not be adequate.

EGFR is not predictive of response. This is in contrast to EGFR targeting agents in other tumor types, such as EGFR status as a predictive biomarker in lung cancer. ${ }^{48}$ Future work may explain the lack of linkage between EGFR overexpressing triple negative breast cancer and EGFR targeting agents. This subgroup of patients currently lacks targeted therapeutic options.

Other potential markers include the serum extracellular domains (ECD) of EGFR and HER2, and tumor tissue analysis of receptor activation by phosphorylation status. Baseline levels of serum HER2/ECD are associated with a poor prognosis. However neither baseline nor serial measurement of ECD HER2 or ECD EGFR has shown predictive capacity. ${ }^{47}$ High baseline p-ErbB2, low Day 21 p-ErbB2 and tumor cell apoptosis on Day 21 have been explored with promise.

A phase I biomarker substudy, in which patients $(\mathrm{n}=33)$ with various tumor types and various lapatinib doses provided a pre-treatment and a sequential Day 21 biopsy, allowed a pilot exploration of the impact of lapatinib on growth and survival pathways. ${ }^{49}$ Four patients with a PR had ErbB2 overexpression and high pre-treatment expression of phosphorylated (p) ErbB2, which was inhibited by lapatinib. Expression of ErbB2 protein was largely unchanged by lapatinib. Clinical response correlated with increased tumor cell apoptosis on Day 21. In contrast, both responders and some nonresponders displayed varying degrees of inhibition of p-ErbB1, pErk1/2, p-Akt and cyclin D1. The nonresponders appeared to have lower baseline levels of ErbB2 and p-ErbB2 than responders. Not all patients with ErbB2 overexpression responded. Indeed, ErbB2 overexpression did not always correlate with an activated receptor as indicated by low levels of p-ErbB2.

In a comparison of baseline tumor blocks and lapatinib response in 65 patients, RT-PCR was used to assess ErbB1-4, PTEN and c-MYC. ${ }^{50}$ Elevated ErbB2 was significantly associated with lapatinib response $(P=0.02)$ and longer TTP $(P<0.0025)$. Of 17 patients with a response, 16 appeared to have a gene expression signature that combined ERBB1, ERBB2 and ERBB3. No association was seen for ErbB4, PTEN or c-MYC.

A retrospective biomarker substudy from the phase III trial of paclitaxel plus lapatinib or placebo explored the potential correlation between hormone subtypes and benefit from lapatinib. ${ }^{51}$ For 493 of the 579 patients, IHC determined semiquantitative ER, progesterone receptor (PgR) and EGFR, and FISH determined HER2 amplification. The subgroups were small, but allowed for exploratory analysis between biomarker expression and EFS. Interestingly, for the HER2positive patients as a group, median EFS was significantly improved ( 8.1 vs 5.0 months; $P=0.008 ; \mathrm{HR}=0.49 ; 95 \% \mathrm{CI}$ 0.3 to 0.8 ). However within the HER2-positive subgroup, statistically significant lapatinib benefit in EFS was not 
seen in patients with coexisting ER or PgR positivity. This analysis lacked statistical power due to the limited sample sizes however even with these patient numbers, heterogeneity within the HER2-positive population is evident. The benefit seen in the HER2-positive, and ER-negative and PgR-negative disease has a strong biological rationale, in that these tumors are dependent on ErbB signaling pathways for survival and progression. Statistically significant findings were incremental benefit from the addition of lapatinib in HER2-positive, ER-negative and PgR-negative disease $(n=42 ; 8.3$ vs 5.0 months; $P=0.007)$ and in HER2-negative, ER-positive and $\mathrm{PgR}$-weakly positive disease $(\mathrm{n}=50 ; 7.3$ vs 2.4 months; $P=0.026)$. In HER2-negative, ER-positive, PgR-negative $\mathrm{MBC}$, lapatinib conferred a worse outcome $(\mathrm{n}=40 ; 3.7$ vs 7.2 months; $P=0.004)$. No significant benefit was seen in the triple negative cancer cohort despite theoretical sensitivity due to increased EGFR expression in this subgroup and the EGFR inhibition by lapatinib. Although most trial patients $(71 \%)$ were negative for EGFR by IHC (EGFR $=0)$, the majority with a positive result had triple negative disease.

\section{Future and place in therapy for lapatinib}

The place for lapatinib in the management of $\mathrm{MBC}$ will be refined with further investigation. Lapatinib is active and well tolerated upfront and in patients pretreated with chemotherapy and trastuzumab. There is biological rationale and clinical evidence to support the use of dual EGFR/HER2-targeted agents in HER-2 positive disease. The role of lapatinib in HER2-negative disease remains unclear. EGFR status has not shown correlation with response. In the first-line management of $\mathrm{MBC}$, prospective data support concurrent use of lapatinib with letrozole in HER2-positive disease. We await results of the phase III trial assessing lapatinib and paclitaxel, with background retrospective analyses favoring the combination therapy in HER2 positive disease.

The optimal duration of lapatinib treatment in MBC is unknown. Uncertainty persists regarding choice between upfront trastuzumab versus lapatinib, dual versus sequential HER2 blockade and best use of lapatinib beyond relapse or progression despite anti-HER2 therapy. There are no clear answers on whether patients with disease relapse following adjuvant trastuzumab are best treated with repeat exposure to trastuzumab or a switch to upfront lapatinib. The disease-ree interval and tolerance may play a role in deciding rechallenge with trastuzumab, additional HER2 blockade with lapatinib or lapatinib alone. Similarly for MBC patients progressing on lapatinib, there is uncertainty regarding subsequent therapy.
A reasonable option would be continued blockade of the HER2 receptor by lapatinib whilst changing the concurrent therapy, however no controlled clinical studies have been reported to currently support such an approach.

Biomarkers for prediction of response, measurement of response and prediction of toxicity are required. Beyond HER2 positive status, refined patient selection is lacking. Monitoring, with valuable tools such as CTC may show treatment efficacy. ${ }^{52}$ Although severe toxicity is uncommon, clinical tools to predict and thus avoid adverse effects would be valuable.

\section{Acknowledgments}

The authors wish to acknowledge the support of the Associazione "Sandro Pitigliani" Prato, Italy and the Associazione Italiana Ricerca Cancro, Milan, Italy.

\section{Disclosures}

The authors disclose no conflicts of interest.

\section{References}

1. Xia W, Mullin RJ, Keith BR, et al. Anti-tumor activity of GW572016: A dual tyrosine kinase inhibitor blocks EGF activation of EGFR/ erbB2 and downstream Erk1/2 and AKT pathways. Oncogene. 2002;21: 6255-6263.

2. Rusnak DW, Lackey K, Affleck K, et al. The effects of the novel, reversible epidermal growth factor receptor/ErbB-2 tyrosine kinase inhibitor, GW572016, on the growth of human normal and tumor-derived cell lines in vitro and in vivo. Mol Cancer Ther. 2001;1:85-94.

3. Lapatinib [package insert].

4. Bence AK, Anderson EB, Doukas MA, et al. Phase I pharmacokinetic studies evaluating single and multiple doses of oral GW572016, a dual EGFR-ErbB2 inhibitor, in healthy subjects. Invest New Drugs. 2005;23:39-49.

5. Versola M, Burris HA, Jones S, et al. Clinical activity of GW572016 in EGF10003 in patients with solid tumors. J Clin Oncol. 2004;14S: abstract 3047

6. Minami H, Nakagawa K, Kawada K, et al. A phase I study of GW572016 in patients with solid tumors. J Clin Oncol. 2004;14S: abstract 3048 .

7. Burris HA, Hurwitz HI, Dees EC, et al. Phase I safety, pharmacokinetics, and clinical activity study of lapatinib (GW572016), a reversible dual inhibitor of epidermal growth factor receptor tyrosine kinases, in heavily pretreated patients with metastatic carcinomas. J Clin Oncol. 2005;23:5305-5313.

8. Medina PJ, Goodin S. Lapatinib: a dual inhibitor of human epidermal growth factor receptor tyrosine kinases. Clin Ther. 2008;30: 1426-1447.

9. LoRusso PM, Jones SF, Koch KM, et al. Phase I and pharmacokinetic study of lapatinib and docetaxel in patients with advanced cancer $J$ Clin Oncol. 2008;18:3051-3056.

10. Storniolo AM, Pegram MD, Overmoyer B, et al. Phase I Dose escalation and pharmacokinetic study of lapatinib in combination with trastuzumab in patients with advanced ErbB2-positive breast cancer. J Clin Oncol. 2008;20:3317-3323.

11. Geyer CE, Forster J, Lindquist D, et al. Lapatinib plus capecitabine for HER2-positive advanced breast cancer. $N$ Engl J Med. 2006;26: $2733-2743$. 
12. Ciccarese M, Lorusso V. How should we prescribe lapatinib to our patients: once daily or twice daily, and at what dose? J Clin Oncol. 2009;2:314-315.

13. Gomez HL, Doval DC, Chavez MA, et al. Efficacy and safety of lapatinib as first-line therapy for ErbB2-amplified locally advanced or metastatic breast cancer. J Clin Oncol. 2008;18:2999-3005.

14. Koch KM, Reddy NJ, Cohen RB, et al. Effects of food on the relative bioavailability of lapatinib in cancer patients. J Clin Oncol. 2009;8: 1191-1196.

15. Burstein HJ, Storniolo AM, Franco S, et al. A phase II study of lapatinib monotherapy in chemotherapy-refractory HER-2 postive and HER2-negative advanced or metastatic breast cancer. Ann Oncol. 2008;6:1068-1074.

16. Blackwell KL, Pegram MD, Tan-Chiu E, et al. Single-agent lapatinib for HER2 overexpressing advanced or metastatic breast cancer that progressed on first- or second-line trastuzumab-containing regimens. Ann Oncol. 2009;206:1026-1033.

17. Di Leo A, Gomez HL, Aziz Z, et al. Phase III, double-blind, randomized study comparing lapatinib plus paclitaxel with placebo plus paclitaxel as first-line treatment for metastatic breast cancer. J Clin Oncol. 2008;34:5544-5552.

18. Johnston S, Pippen P, Pivot X, et al. Lapatinib combined with letrozole versus letrozole and placebo as first-line therapy for postmenopausal hormone receptor-positive metastatic breast cancer. J Clin Oncol. 2009;27:5538-5546.

19. Wolff A, Hammond M, Schwartz J, et al. American Society of Clinical Oncology/College of American Pathologists guideline recommendations for human epidermal growth factor receptor 2 testing in breast cancer. J Clin Oncol. 2007;1:118-145.

20. Simmons C, Miller N, Geddie W, et al. Does confirmatory tumor biopsy alter the management of breast cancer patients with distant metastases? Ann Oncol. 2009;20: 499-504.

21. Meng S, Tripathy D, Shete S, et al. HER-2 gene amplification can be acquired as breast cancer progresses. Proc Natl Acad Sci U S A. 2004; 10125:9393-9398.

22. Pestrin M, Bessi S, Galardi F, et al. Correlation of HER2 status between primary tumors and corresponding circulating tumor cells in advanced breast cancer patients. Breast Cancer Res Treat. 2009;118: 523-530.

23. O’Shaughnessy J, Blackwell KL, Burstein H, et al. A randomized study of lapatinib alone or in combination with trastuzumab in heavily pretreated HER2 + metastatic breast cancer progressing on trastuzumab therapy. J Clin Oncol. 2008;26 Suppl: abstract 1015.

24. Koch KM, Lee D, Jones S, et al. Pharmacokinetics of GW572016 in an ascending dose tolerability study of phase I cancer patients. Eur J Cancer. 2003;1 Suppl:S169.

25. Baselga J, Carbonell X, Castaneda-Soto NJ, et al. Phase II study of efficacy, safety, and pharmacokinetics of trastuzumab monotherapy administered on a 3-weekly schedule. J Clin Oncol. 2005;10:2162-2171.

26. Vogel CL, Cobleigh MA, Tripathy D, et al. Efficacy and safety of trastuzumab as a single agent in first-line treatment of HER2-overexpressing metastatic breast cancer. J Clin Oncol. 2002;3:719-726.

27. Jones SF, Hainsworth JD, Spigel DR, et al. A phase I study of the dual kinase inhibitor GW572016 in combination with paclitaxel (EGF10009). J Clin Oncol. 2004;22:14S: abstract 2083.

28. Gee JM, Harper ME, Hutcheson IR, et al. The antiepidermal growth factor receptor agent gefitinib (ZD1839/Iressa) improves antihormone response and prevents development of resistance in breast cancer in vitro. Endocrinology. 2003;144:5105-5117.

29. Mackey JR, Kaufman B, Clemens M, et al. Trastuzumab prolongs progression free survival in hormone-dependent and HER2-positive metastatic breast cancer. Breast Cancer Res Treat. 2006;100: abstract 3.

30. Cristofanilli M, Valero V, Mangalik A, et al. A phase II multicenter, double-blind, randomized trial to compare anastrozole plus gefinitib with anastrozole plus placebo in postmenopausal women with hormone receptor-positive (HR+) metastatic breast cancer (MBC). J Clin Oncol. 2008;26 Suppl: abstract 1012.
31. Osborne K, Neven P, Dirix L, et al. Randomised phase II study of gefitinib (IRESSA) or placebo in combination with tamoxifen in patients with hormone receptor positive metastatic breast cancer. Breast Cancer Res Treat. 2007;106 Suppl 1:S107.

32. Gril B, Palmieri D, Bronder JL, et al. Effect of Lapatinib on the Outgrowth of Metastatic Breast Cancer Cells to the Brain. J Natl Cancer Inst. 2008;15:1092-1103.

33. Lin NU, Carey LA, Liu MC, et al. Phase II trial of lapatinib for brain metastases in patients with human epidermal growth factor receptor 2-positive breast. Cancer J Clin Oncol. 2008;12:1993-1999.

34. Lin NU, Dieras V, Paul D, et al. EGF105084, a phase II study of lapatinib for brain metastases in patients (pts) with HER2+ breast cancer following trastuzumab $(\mathrm{H})$ based systemic therapy and cranial radiotherapy (RT). J Clin Oncol. 2007;25:18S: abstract 1012.

35. Lin NU, Paul D, Dieras V, et al. Lapatinib and capecitabine for the treatment of brain metastases in patients with HER2+ breast cancer an updated analysis from EGF105084. San Antonio Breast Cancer Symposium; 2007: abstract 6076.

36. Crown JP, Burris HA, Boyle F, et al. Pooled analysis of diarrhea events in patients with cancer treated with lapatinib. Breast Cancer Res Treat. 2008;2:317-325.

37. Lacouture ME, Laabs SM, Koehler M, et al. Analysis of dermatologic events in patients with cancer treated with lapatinib. Breast Cancer Res Treat. 2009;3:485-493.

38. Perez E, Byrne J, Hammond I, et al. Cardiac safety experience in 3127 patients treated with lapatinib. Ann Oncol. 2006;17(Suppl 9)ix70: abstract 1420

39. Robinson AS, Ellard S, Speers C, et al. Clinical and molecular predictors of sustained response to trastuzumab in metastatic breast cancer. J Clin Oncol. 2005;23:16S: abstract 500.

40. Pegram M. Can we circumvent resistance to ErbB2-targeted agents by targeting novel pathways? Clin Breast Cancer. 2008;8:Suppl 3: S121-S130.

41. Xia W, Liu LH, Ho P, et al. Truncated erbB2 receptor (p95erbB2) is regulated by heregulin through heterodimer formation with erbB3 yet remains sensitive to the dual EGFR/ErbB2 kinase inhibitor GW572016. Oncogene. 2004;23:646-653.

42. Nagata Y, Lan KH, Zhou X, et al. PTEN activation contributes to tumor inhibition by trastuzumab, and loss of PTEN predicts trastuzumab resistance in patients. Cancer Cell. 2004;2:117-127.

43. Xia W, Husain I, Liu L, et al. Lapatinib antitumor activity is not dependent upon phosphatase and tensin homologue deleted on chromosome 10 in ErbB2-overexpressing breast cancers. Cancer Res. 2007;3:1170-1175.

44. Arpino G, Wiechmann L, Osborne CK, et al. Crosstalk between the estrogen receptor and the HER tyrosine kinase receptor family: Molecular mechanism and clinical implications for endocrine therapy resistance. Endocr Rev. 2008;29:217-233.

45. Chen FL, Xia W, Spector NL. Acquired resistance to small molecule ErbB2 tyrosine kinase inhibitors. Clin Cancer Res. 2008;21: 6730-6734.

46. Zhou X, Cella D, Cameron D, et al. Lapatinib plus capecitabine versus capecitabine alone for HER2+ $(\mathrm{ERbB} 2+)$ metastatic breast cancer: quality of life assessment. Breast Cancer Res Treat. 2009;3: 577-589.

47. Cameron D, Casey M, Press M, et al. A phase III randomized comparison of lapatinib plus capecitabine versus capecitabine alone in women with advanced breast cancer that has progressed on trastuzumab: updated efficacy and biomarker analyses. Breast Cancer Res Treat. 2008;3:533-543.

48. Kobayashi S, Boggon TJ, Dayaram T, et al. EGFR mutation and resistance of non-small-cell lung cancer to gefitinib. $N$ Engl J Med. 2005;352:786-792.

49. Spector NL, Xia W, Burris H III, et al. Study of the biological effects of lapatinib, a reversible inhibitor of ErbB1 and ErbB2 tyrosine kinases, on tumor growth and survival pathways in patients with advanced malignancies. J Clin Oncol. 2005;11:1-11. 
50. Gomez HL, Chavez DC, Doval DC, et al. Investigation of tumor biomarkers as response predictors in a monotherapy stucy with lapatinib (L) as a first line treatment in ErbB2 amplified women with breast cnacer. J Clin Oncol. 2007;25:18S: abstract 10562.

51. Finn RS, Press MF, Dering J, et al. Estrogen receptor, progesterone receptor, human epidermal growth factor 2 (HER2), and epidermal growth factor receptor expression and benefit from lapatinib in a randomised trial of paclitaxel with lapatinib or placebo as first-line treatment in HER2-negative or unknown metastatic breast cancer. J Clin Oncol. 2009;24:3908-3915.
52. Camara O, Jörke C, Hammer U, et al. Monitoring circulating epithelial tumour cells (CETC) to gauge therapy: in patients with disease progression after trastuzumab persisting CETC can be eliminated by combined lapatinib treatment. J Cancer Res Clin Oncol. 2009;4: 643-647.

\section{Publish your work in this journal}

Cancer Management and Research is an international, peer-reviewed open access journal focusing on cancer research and the optimal use of preventative and integrated treatment interventions to achieve improved outcomes, enhanced survival and quality of life for the cancer patient The journal welcomes original research, clinical \& epidemiological studies, reviews \& evaluations, guidelines, expert opinion \& commentary, case reports \& extended reports. The manuscript management system is completely online and includes a very quick and fair peerreview system, which is all easy to use. Visit http://www.dovepress.com/ testimonials.php to read real quotes from published authors.

Submit your manuscript here: http://www.dovepress.com/cancer-management-and-research-journal 